\title{
Lateral control strategy for autonomous steering of Ackerman-like vehicles
}

\author{
Miguel Angel Sotelo* \\ Department of Electronics, Escuela Politécnica, University of Alcalá, Campus Universitario s/n, \\ 28871 Alcalá de Henares, Madrid, Spain
}

Received 18 November 2002; received in revised form 1 September 2003; accepted 22 September 2003

\begin{abstract}
This paper presents the results of a lateral control strategy that has been applied to the problem of steering an autonomous vehicle using vision. The lateral control law has been designed for any kind of vehicle presenting the Ackerman kinematic model, accounting for the vehicle velocity as a crucial parameter for adapting the steering control response. This makes the control strategy suitable for either low or high speed vehicles. The stability of the control law has been analytically proved, and experimentally tested by autonomously steering Babieca, a Citroën Berlingo prototype vehicle.
\end{abstract}

(c) 2003 Elsevier B.V. All rights reserved.

Keywords: Lateral control strategy; Non-linear steering kinematics; Autonomous Ackerman-like vehicles

\section{Introduction}

Lateral automatic steering of autonomous Ackerman-like vehicles has become an apparent field of application for robotics researchers, due to the increasing speed of modern processors. Basically, this problem can be stated as that of determining an appropriate control law for commanding the vehicle steering angle. Several research groups have already demonstrated impressive results on this control task [9-11]. Thus, many steering control designs are documented in the literature $[1,2,5,12,13,16]$. A comparative study on various lateral control strategies for autonomous vehicles can be found in [14,19], where a linearized model of the lateral vehicle dynamics is used for controller design basing on the fact that it is possible to decouple the longitudinal and lateral dynamics.

On the contrary, a simplified non-linear lateral kinematic model is proposed in this work, intended to ease the design and implementation of a stable lateral control law for autonomous steering of Ackerman-like vehicles. The lateral control strategy was implemented on Babieca, an electric Citroên Berlingo experimental prototype, using vision as the main sensor to measure the position of the vehicle in the road. Real tests were carried out on a private circuit emulating an urban quarter, composed of streets, intersections (crossroads), and roundabouts, located at the Industrial Automation Institute (IAI) in Arganda del Rey, Madrid. Additionally, a live demonstration exhibiting

\footnotetext{
* Tel.: +34-91-885-6573; fax: +34-91-885-6591.
}

E-mail address: michael@depeca.uah.es (M.A. Sotelo). 
the system capacities on autonomous steering was carried out during the IEEE Conference on Intelligent Vehicles 2002, in a private circuit located at Satory (Versailles), France.

The work described in this paper is organized in the following sections: Section 2 provides a description of the complete lateral control strategy developed for autonomous steering of Ackerman-like vehicles, as well as stability demonstrations. Section 3 describes the system implementation and control results, while, finally, a discussion including conclusions and the future work to be carried out is described in Section 4.

\section{Lateral control}

Considering the case of an autonomous vehicle driving along some reference trajectory, the main goal of the lateral control module is to ensure proper tracking of the reference trajectory by correctly keeping the vehicle in the center of the lane with the appropriate orientation (parallel to the desired trajectory). This constraint can be generalized as the minimization of the vehicle lateral and orientation errors $\left(d_{\mathrm{e}}, \theta_{\mathrm{e}}\right)$ at a given distance $L_{\mathrm{h}}$, denoted as look-ahead distance, with respect to the reference trajectory. Graphically, the problem is depicted in Fig. 1 where the vehicle control point has been located at the look-ahead distance $L_{\mathrm{h}}$ along the vehicle longitudinal axis in order to properly anticipate the trajectory curvature. The choice of $L_{\mathrm{h}}$ is discussed in Section 2.1. To solve this controllability problem and design a stable lateral controller, a model describing the dynamic behavior of $d_{\mathrm{e}}$ and $\theta_{\mathrm{e}}$ is needed.

\subsection{Kinematic model}

The kinematic model of the vehicle is the starting point to model the dynamics of the lateral and orientation errors. The vehicle model is approximated by the popular Ackerman (or bicycle) model [6] as depicted in Fig. 2, assuming that the two front wheels turn slightly differentially and thus, the instantaneous rotation center can be purely computed by kinematic means. Let $\kappa(t)$ denote the instantaneous curvature of the trajectory described by the vehicle:

$$
\kappa(t)=\frac{1}{R(t)}=\frac{\tan \phi(t)}{L}=\frac{\mathrm{d} \theta(t)}{\mathrm{d} s},
$$

where $R$ is the radius of curvature, $L$ the wheelbase, $\phi$ the steering angle, $s$ the path length, and $\theta$ the vehicle orientation in a global frame of coordinates. The dynamics of $\theta$ are computed in Eq. (2) as a function of vehicle

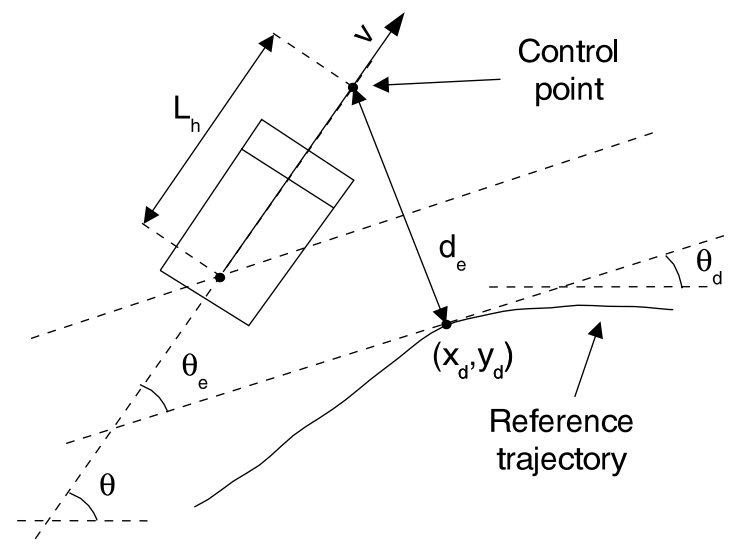

Fig. 1. Lateral and orientation errors at the look-ahead distance with respect to a reference trajectory. 


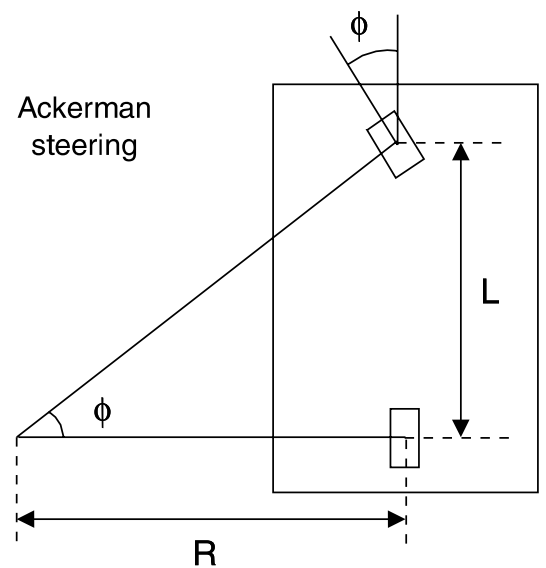

Fig. 2. Approximate kinematic model of the vehicle (Ackerman steering).

velocity $v$ :

$$
\dot{\theta}=\frac{\mathrm{d} \theta}{\mathrm{d} t}=\frac{\mathrm{d} \theta}{\mathrm{d} s} \cdot \frac{\mathrm{d} s}{\mathrm{~d} t}=\kappa(t) \cdot v(t)=\frac{\tan \phi(t)}{L} \cdot v(t) .
$$

Let $\phi$ and $v$ be the variables of the vehicle actuation space. On the other hand, the vehicle configuration space is composed of the global position and orientation variables, described by $(x, y, \theta)$, under the flat terrain assumption. Mapping from the actuation space to the configuration space can be solved by using the popular Fresnel equations, which are also the so-called dead reckoning equations typically used in inertial navigation. Eq. (3) shows the dynamics of $(x, y, \theta)$ :

$$
\dot{x}=\frac{\mathrm{d} x}{\mathrm{~d} t}=v(t) \cos \theta(t), \quad \dot{y}=\frac{\mathrm{d} y}{\mathrm{~d} t}=v(t) \sin \theta(t), \quad \dot{\theta}=\frac{\mathrm{d} \theta}{\mathrm{d} t}=v(t) \frac{\tan \phi(t)}{L},
$$

where $v(t)$ represents the velocity of the midpoint of the vehicle rear axle, which can be considered to be the same as that of the control point at the look-ahead distance. Global information about the position and orientation of the vehicle $(x, y, \theta)$ is then transformed so as to develop a model that describes the open-loop lateral and orientation error dynamics. As observed in Fig. 1, the lateral error $d_{\mathrm{e}}$ is defined as the distance between the vehicle control point and the closest point along the vehicle desired trajectory, described by coordinates $\left(x_{\mathrm{d}}, y_{\mathrm{d}}\right)$. This implies that $d_{\mathrm{e}}$ is perpendicular to the tangent to the reference trajectory at $\left(x_{\mathrm{d}}, y_{\mathrm{d}}\right)$. The slope of the tangent at $\left(x_{\mathrm{d}}, y_{\mathrm{d}}\right)$ is denoted by $\theta_{\mathrm{d}}$, and represents the desired orientation at that point. Based on this, $d_{\mathrm{e}}$ and $\theta_{\mathrm{e}}$ suffice to precisely characterize the location error between the vehicle and some given reference trajectory, as described in Eq. (4):

$$
d_{\mathrm{e}}=-\left(x+L_{\mathrm{h}} \cos \theta-x_{\mathrm{d}}\right) \sin \theta_{\mathrm{d}}+\left(y+L_{\mathrm{h}} \sin \theta_{\mathrm{d}}-y_{\mathrm{d}}\right) \cos \theta_{\mathrm{d}}, \quad \theta_{\mathrm{e}}=\theta-\theta_{\mathrm{d}} .
$$

The choice of $L_{\mathrm{h}}$ is carried out based on the current vehicle velocity $v$, yielding the parameters shown in Eq. (5). It has been demonstrated [4] that, as $v$ increases, the damping factor of the closed loop system gets worse and can be improved, under certain limits, by increasing the look-ahead distance:

$$
L_{\mathrm{h}}(v)= \begin{cases}L_{\min } & \text { if } v<v_{\min }, \\ v \cdot t_{1} & \text { if } v_{\min } \leq v \leq v_{\max }, \\ L_{\max } & \text { if } v>v_{\max },\end{cases}
$$

where $t_{1}=1.5 \mathrm{~s}$ is the look-ahead time, $v_{\min }=25 \mathrm{~km} / \mathrm{h}, v_{\max }=75 \mathrm{~km} / \mathrm{h}, L_{\min }=10.41 \mathrm{~m}$, and $L_{\max }=31.25 \mathrm{~m}$, empirically determined in our experiments. Computing the derivative of $d_{\mathrm{e}}$ and $\theta_{\mathrm{e}}$ with respect to time yields the 
non-linear model formulated in Eq. (6). In this model, $x_{\mathrm{d}}, y_{\mathrm{d}}$, and $\theta_{\mathrm{d}}$ are calculated at each iteration by a vision-based process, and taken as reference values that do not change as a function of time between two consecutive steps of the control algorithm:

$$
\dot{d}_{\mathrm{e}}=v \sin \theta_{\mathrm{e}}+\frac{v L_{\mathrm{h}}}{L} \cos \theta_{\mathrm{e}} \tan \phi, \quad \dot{\theta}_{\mathrm{e}}=\frac{v \tan \phi}{L} .
$$

\subsection{Non-linear control law}

The control objective is to ensure that the vehicle will correctly track the reference trajectory. For this purpose, both the lateral error $d_{\mathrm{e}}$ and the orientation error $\theta_{\mathrm{e}}$ must be minimized. On the other hand, vehicle velocity $v$ will be assumed to be constant, for simplicity. The design of the control law is based on general results in the so-called chained systems theory [15]. An excellent example on this topic can be found in [7]. Nevertheless, these results are extended and generalized in this paper so as to provide a stable non-linear control law for steering of Ackerman-like vehicles based on local errors. From the control point of view, the use of the popular tangent linearization approach is avoided as it is only valid locally around the configuration chosen to perform the linearization, and thus, the initial conditions may be far away from the reference trajectory. On the contrary, some state and control variables changes are posed in order to convert the non-linear system described in Eq. (6) into a linear one, without any approximation (exact linearization approach). Nevertheless, due to the impossibility of exactly linearizing systems describing mobile robots dynamics, these non-linear systems can be converted in almost linear ones, termed as chained form. The use of the chained form permits to design a control law using linear systems theory to a high extent. In particular, the non-linear model for $d_{\mathrm{e}}$ and $\theta_{\mathrm{e}}$ (Eq. (6)) can be transformed into chained form using the state diffeomorphism and change of control variables, as in Eq. (7):

$$
Y=\left[\begin{array}{l}
y_{1} \\
y_{2}
\end{array}\right]=\Theta(X)=\left[\begin{array}{c}
d_{\mathrm{e}} \\
\tan \theta_{\mathrm{e}}
\end{array}\right], \quad W=\left[\begin{array}{l}
w_{1} \\
w_{2}
\end{array}\right]=\Upsilon(U)=\left[\begin{array}{c}
v \cos \theta_{\mathrm{e}}+\frac{v L_{h} \cos ^{2} \theta_{\mathrm{e}} \tan \phi}{L \sin \theta_{\mathrm{e}}} \\
\frac{v \tan \phi}{L \cos ^{2} \theta_{\mathrm{e}}}
\end{array}\right] .
$$

These transformations are invertible whenever the vehicle speed $v$ is different from zero, and the orientation error $\theta_{\mathrm{e}}$ is different from $\pi / 2$. This implies that the singularities of the transformations can be avoided by assuring that the vehicle moves $(v>0)$ and that its orientation error is maintained under $90^{\circ}$ (the vehicle orientation must not be perpendicular to the reference trajectory). These conditions are reasonably simple to meet in practice. From Eq. (7) the vehicle model can be rewritten as in Eq. (8), considering $y_{1}$ and $y_{2}$ as the new state variables:

$$
\dot{y}_{1}=\dot{d}_{\mathrm{e}}=v \sin \theta_{\mathrm{e}}+\frac{v L_{\mathrm{h}}}{L} \cos \theta_{\mathrm{e}} \tan \phi=w_{1} y_{2}, \quad \dot{y}_{2}=\frac{\mathrm{d}\left(\tan \theta_{\mathrm{e}}\right)}{\mathrm{d} t}=\frac{1}{\cos ^{2} \theta_{\mathrm{e}}} \cdot \dot{\theta}_{\mathrm{e}}=\frac{v \tan \phi}{L \cos ^{2} \theta_{\mathrm{e}}}=w_{2} .
$$

In order to get a velocity-independent control law, the time derivative is replaced by a derivation with respect to $\varsigma$, a variable that is related to the path length described by the vehicle in parallel to the tangent to the reference trajectory. Analytically, $\varsigma$ is computed as expressed in Eq. (9):

$$
\varsigma=\int\left(v \cos \theta_{\mathrm{e}}+\frac{v L_{\mathrm{h}} \cos ^{2} \theta_{\mathrm{e}} \tan \phi}{L \sin \theta_{\mathrm{e}}}\right) \mathrm{d} t .
$$

The time derivative of the state variables $y_{1}$ and $y_{2}$ is expressed as a function of $\varsigma$ in Eq. (10):

$$
\dot{y}_{1}=\frac{\mathrm{d} y_{1}}{\mathrm{~d} t}=\frac{\mathrm{d} y_{1}}{\mathrm{~d} \varsigma} \cdot \frac{\mathrm{d} \varsigma}{\mathrm{d} t}=y_{1}^{\prime} \cdot \dot{\zeta}, \quad \dot{y}_{2}=\frac{\mathrm{d} y_{2}}{\mathrm{~d} t}=\frac{\mathrm{d} y_{2}}{\mathrm{~d} \varsigma} \cdot \frac{\mathrm{d} \varsigma}{\mathrm{d} t}=y_{2}^{\prime} \cdot \dot{\zeta},
$$

where $y_{1}^{\prime}$ and $y_{2}^{\prime}$ stand for the derivative of $y_{1}$ and $y_{2}$ with respect to $\varsigma$. Solving for $y_{1}^{\prime}$ and $y_{2}^{\prime}$ yields Eq. (11):

$$
y_{1}^{\prime}=\frac{\dot{y}_{1}}{\dot{\zeta}}=\tan \theta_{\mathrm{e}}=y_{2}, \quad y_{2}^{\prime}=\frac{\dot{y}_{2}}{\dot{\zeta}}=\frac{\tan \phi}{L \cos ^{3} \theta_{\mathrm{e}}+L_{\mathrm{h}}\left(\cos ^{4} \theta_{\mathrm{e}} \tan \phi / \sin \theta_{\mathrm{e}}\right)}=\frac{w_{2}}{w_{1}}=w_{3} .
$$


As observed in the previous equation, the transformed system is linear and thus, state variables $y_{1}$ and $y_{2}$ can be regulated to zero ( so as to yield $d_{\mathrm{e}}=d_{\mathrm{e}, \text { ref }}=0$ and $\theta_{\mathrm{e}}=\theta_{\mathrm{e}, \text { ref }}=0$ ) by using the control law proposed in Eq. (12):

$$
w_{3}=-K_{\mathrm{d}} y_{2}-K_{\mathrm{p}} y_{1}, \quad\left(K_{\mathrm{d}}, K_{\mathrm{p}}\right) \in \mathfrak{R}^{+2} .
$$

It must be remarked that through this choice many degrees of freedom for control selection are lost. Nonetheless, the results are acceptable as will be demonstrated in Section 3. Using Eqs. (11) and (12) and solving for variable $y_{1}$ yields Eq. (13), where the dynamic behavior of $y_{1}$ with respect to $\varsigma$ is demonstrated to be linear:

$$
y_{1}^{\prime \prime}+K_{\mathrm{d}} y_{1}^{\prime}+K_{\mathrm{p}} y_{1}=0 .
$$

This implies that variables $y_{1}=d_{\mathrm{e}}$ and $y_{2}=\tan \theta_{\mathrm{e}}$ tend to zero as variable $\varsigma$ grows. The previous statement is analytically expressed in Eq. (14):

$$
\lim _{\varsigma \rightarrow \infty} d_{\mathrm{e}}=\lim _{\varsigma \rightarrow \infty} \theta_{\mathrm{e}}=0 .
$$

Accordingly, variable $\varsigma$ must always grow so as to ensure that both $d_{\mathrm{e}}$ and $\theta_{\mathrm{e}}$ tend to zero. This condition is met whenever $v>0$ and $-\pi / 2<\theta_{\mathrm{e}}<\pi / 2$. In other words, the vehicle must continuously move forward and the absolute value of its orientation error should be below $\pi / 2$ in order to guarantee proper trajectory tracking. Thus, the non-linear control law is finally derived in Eq. (15) from Eqs. (11) and (12):

$$
\phi=\arctan \left[\frac{-L \sin \theta_{\mathrm{e}} \cos ^{3} \theta_{\mathrm{e}}\left(K_{\mathrm{d}} \tan \theta_{\mathrm{e}}+K_{\mathrm{p}} d_{\mathrm{e}}\right)}{\sin \theta_{\mathrm{e}}+L_{\mathrm{h}} \cos ^{4} \theta_{\mathrm{e}}\left(K_{\mathrm{d}} \tan \theta_{\mathrm{e}}+K_{\mathrm{p}} d_{\mathrm{e}}\right)}\right] .
$$

The control law is then modified by a sigmoidal function as shown in Eq. (16), to account for physical limitations in the vehicle wheels turning angle and prevent from actuator saturation. On the other hand, the use of sigmoidal functions preserves the system stability [18]:

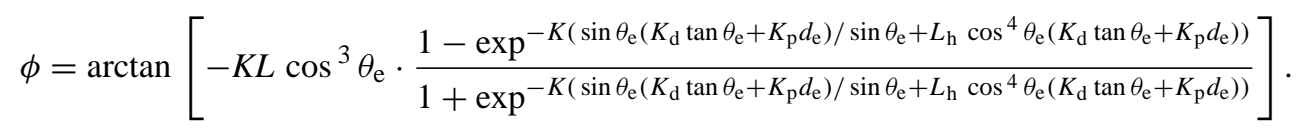

The control law is saturated to $\phi_{\max }$ by properly tuning parameter $K$. Thus, the maximum value of Eq. (16) is $\phi_{\max }= \pm \arctan (-K L)$. Therefore, $K$ is chosen to ensure that $\phi_{\max }= \pm(\pi / 6)$ rad (physical limitation of the vehicle), given the wheelbase $L=2.69 \mathrm{~m}$, yielding a practical value $K=0.2146$ :

$$
K=\frac{\tan (\pi / 6)}{L} .
$$

From the observation of Eq. (13), the dynamic response of variable $y_{1}$ can be considered to be a second order linear one. In practice, it is not indeed linear due to the sigmoidal function used to saturate the control law, although it can be reasonably approximated as such. Thus, an analogy between constants $K_{\mathrm{d}}, K_{\mathrm{p}}$, and the parameters of a second order linear system $\xi$ (damping coefficient) and $\omega_{\mathrm{n}}$ (natural frequency) can be established, yielding Eq. (18):

$$
\omega_{\mathrm{n}}=\sqrt{K_{\mathrm{p}}}, \quad \xi=\frac{K_{\mathrm{d}}}{2 \sqrt{K_{\mathrm{p}}}} .
$$

Likewise, system overshoot $M_{\mathrm{p}}$ and settling distance $d_{\mathrm{s}}$ (given that the system error dynamics are described as a function of space variable $\zeta$, not time) can be obtained from Eq. (19):

$$
M_{\mathrm{p}}=\exp \left(\frac{-\xi \pi}{\sqrt{1-\xi^{2}}}\right), \quad d_{s \mid 2 \%}=\frac{4}{\xi \omega_{\mathrm{n}}} .
$$


The design of constants $K_{\mathrm{d}}$ and $K_{\mathrm{p}}$ is undertaken considering that the system overshoot must not exceed $10 \%$ of the step input, and that the settling distance should be below some given threshold. Thus, for a typical settling time $t_{\mathrm{s}}=20 \mathrm{~s}$, and given the vehicle velocity $v$, the proper settling distance can be computed as in Eq. (20):

$$
d_{\mathrm{s}}=t_{\mathrm{s}} \cdot v=20 v \text {. }
$$

The value of $K_{\mathrm{d}}$ is derived from Eqs. (18) and (19) yielding the velocity dependant expression in Eq. (21):

$$
K_{\mathrm{d}}=\frac{8}{d_{\mathrm{s}}}=\frac{0.4}{v} .
$$

Likewise, damping coefficient $\xi$ is derived from Eqs. (18) and (19), as shown in Eq. (22):

$$
\xi=\sqrt{\frac{1}{[\pi / \ln 0.1]^{2}+1}}=\frac{K_{\mathrm{d}}}{2 \sqrt{K_{\mathrm{p}}}}=\frac{4}{d_{\mathrm{s}} \sqrt{K_{\mathrm{p}}}} .
$$

Finally, $K_{\mathrm{p}}$ is deduced from the previous equation, yielding Eq. (23):

$$
K_{\mathrm{p}}=\left[\frac{6.766}{d_{\mathrm{s}}}\right]^{2}=\left[\frac{0.3383}{v}\right]^{2} .
$$

The dependence of $K_{\mathrm{p}}$ and $K_{\mathrm{d}}$ on vehicle velocity $v$ permits to ensure proper dynamic response. In particular, vehicle turning angle will be soft at high speeds, therefore avoiding possible oscillations due to physical constraints in steering dynamics.

\section{Implementation and results}

The control law for autonomous steering described in this paper was tested on the so-called Babieca prototype vehicle (an electric Citroén Berlingo), as depicted in Fig. 3. The vehicle was modified to allow for automatic velocity and steering control at a maximum speed of $90 \mathrm{~km} / \mathrm{h}$. Babieca is equipped with a color camera to provide lateral and orientation position of the ego-vehicle with regard to the center of the lane, a DGPS receiver, a Pentium PC, and a set of electronic devices to provide actuation over the accelerator and steering wheel, as well as to encode the vehicle velocity and steering angle. The color camera provides standard PAL video signal at $25 \mathrm{~Hz}$ that is processed by a Meteor frame grabber installed on a $120 \mathrm{MHz}$ Pentium running the Real Time Linux operating system. On the other hand, the DGPS receiver is a Z-12 Real Time model by Ashtech that implements the RTCM SC 104 V2.2 standard. The complete navigation system, implemented under Real Time Linux using a pre-emptive scheduler [3], runs a vision-based lane tracking task for computing the lateral and orientation errors, as described in [17].

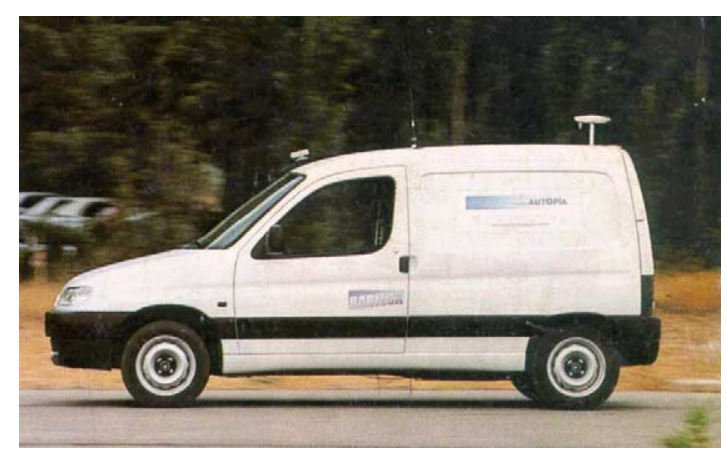

Fig. 3. Babieca prototype vehicle. 


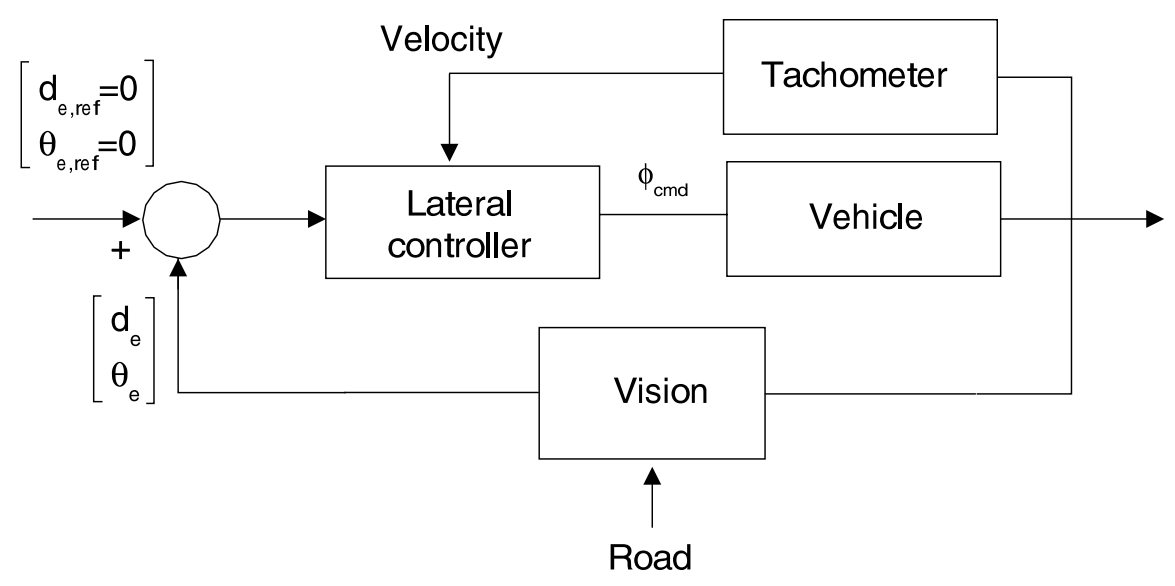

Fig. 4. Close-loop lateral control scheme.

Practical experiments were conducted on a private circuit located at the Industrial Automation Institute in Arganda del Rey (Madrid). The circuit is composed of several streets, intersections, and roundabout points, trying to emulate an urban quarter. The complete close-loop scheme implemented for lateral control of the test vehicle is depicted in Fig. 4. As can be observed, the control objective is to achieve the reference error vector $d_{\mathrm{e}, \text { ref }}=0$ and $\theta_{\mathrm{e} \text {, ref }}=0$. This objective implies proper tracking of the road curvature perceived by the vision system.

Various practical trials were conducted so as to test the validity of the control law for different initial conditions in real circumstances. During the tests, the reference vehicle velocity is assumed to be kept constant by a velocity controller developed in [8]. Constants $K_{\mathrm{d}}$ and $K_{\mathrm{p}}$ were calculated as a function of $v$ using Eqs. (21) and (23). In the experiments, a quasi-straight reference path was used to autonomously guide the vehicle based on visual measurements. Figs. 5-7 show the transient response of the vehicle lateral and orientation errors for reference velocities of 10,20 , and $50 \mathrm{~km} / \mathrm{h}$, respectively. In all cases, the vehicle starts the run at an initial lateral error of about $1 \mathrm{~m}$, and an initial orientation error in the range of $\pm 5^{\circ}$. For illustrative purposes, Fig. 8 depicts the steering control provided by the controller during the path tracking experiment carried out at $50 \mathrm{~km} / \mathrm{h}$. As can be clearly appreciated, the steady-state response of the system is satisfactory for the three experiments. Thus, the lateral error
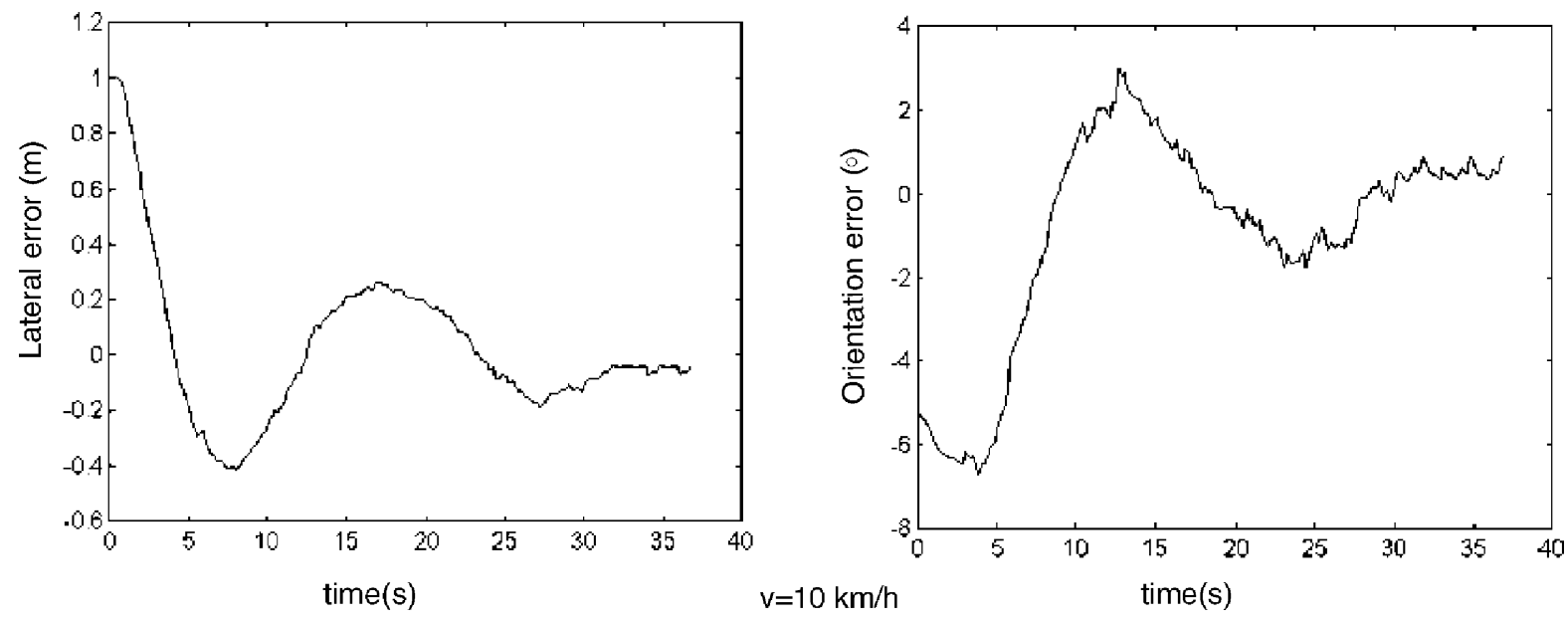

Fig. 5. Transient response of the lateral and orientation error for $v=10 \mathrm{~km} / \mathrm{h}$. 

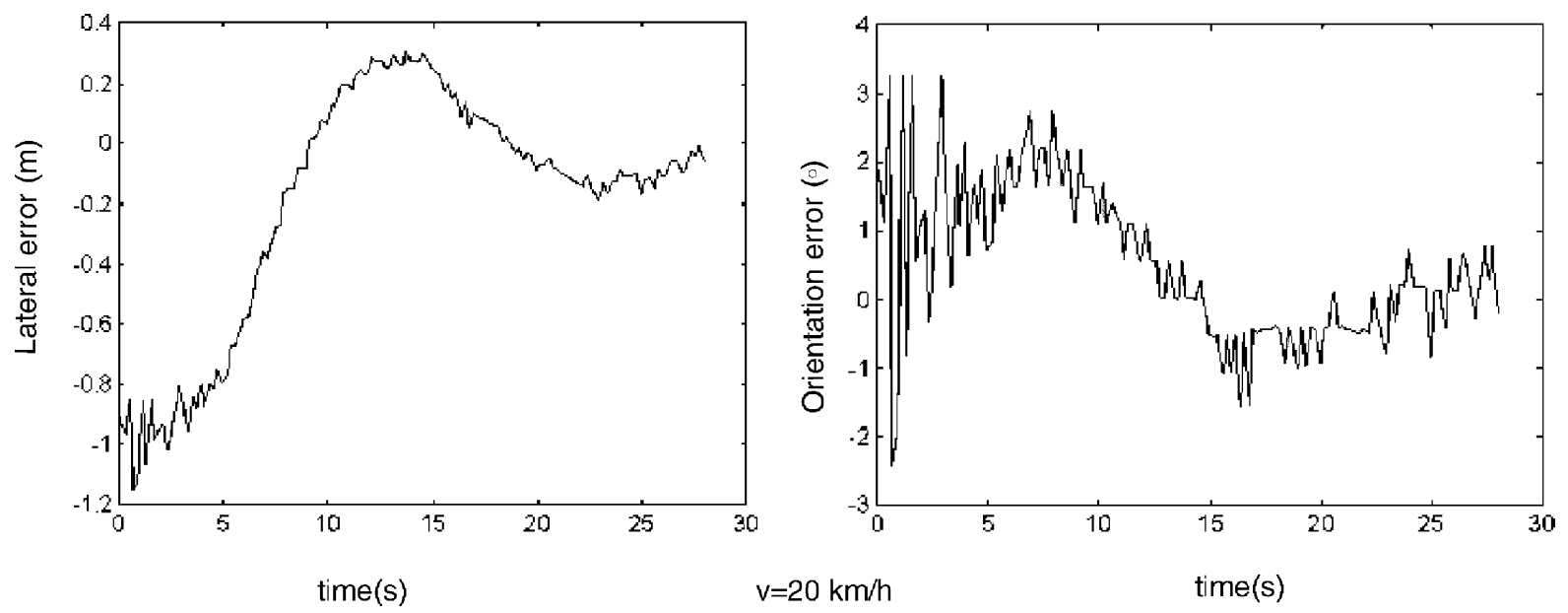

Fig. 6. Transient response of the lateral and orientation error for $v=20 \mathrm{~km} / \mathrm{h}$.

is bound to $\pm 5 \mathrm{~cm}$ at low speeds and $\pm 25 \mathrm{~cm}$ at $v=50 \mathrm{~km} / \mathrm{h}$, while the absolute orientation error in steady state remains below $1^{\circ}$ in all cases. Just to give an example on how the practical results conform to the expected values as derived from the theoretical development, let us consider the transient response of the vehicle depicted in Fig. 6 for $v=20 \mathrm{~km} / \mathrm{h}$. Assuming a theoretical maximum overshoot of $M_{\mathrm{p}}=10 \%$ and a settling time of $t_{\mathrm{s}}=20 \mathrm{~s}$, the controller coefficients are tuned to $K_{\mathrm{d}}=0.072$ and $K_{\mathrm{p}}=0.0037$, according to Eqs. (21) and (23). Nonetheless, from observation of Fig. 6 the maximum overshoot obtained in practice is almost $25 \%$ for both the lateral and orientation errors, while the settling time takes some $22 \mathrm{~s}$. This is mainly due to the existence of non-linear actuator dynamics and latencies, not considered in the model. In spite of these slight differences with regard to the theoretical expected values, the practical results exhibited in this section demonstrate that the non-linear lateral control law developed in this work still permits to safely steer the vehicle at operational velocities.

In a final trial, the results achieved in the second test for $v=20 \mathrm{~km} / \mathrm{h}$ are compared to human driving at the same speed along the same trajectory. For this purpose a human driver steered the vehicle, leaving the control of the
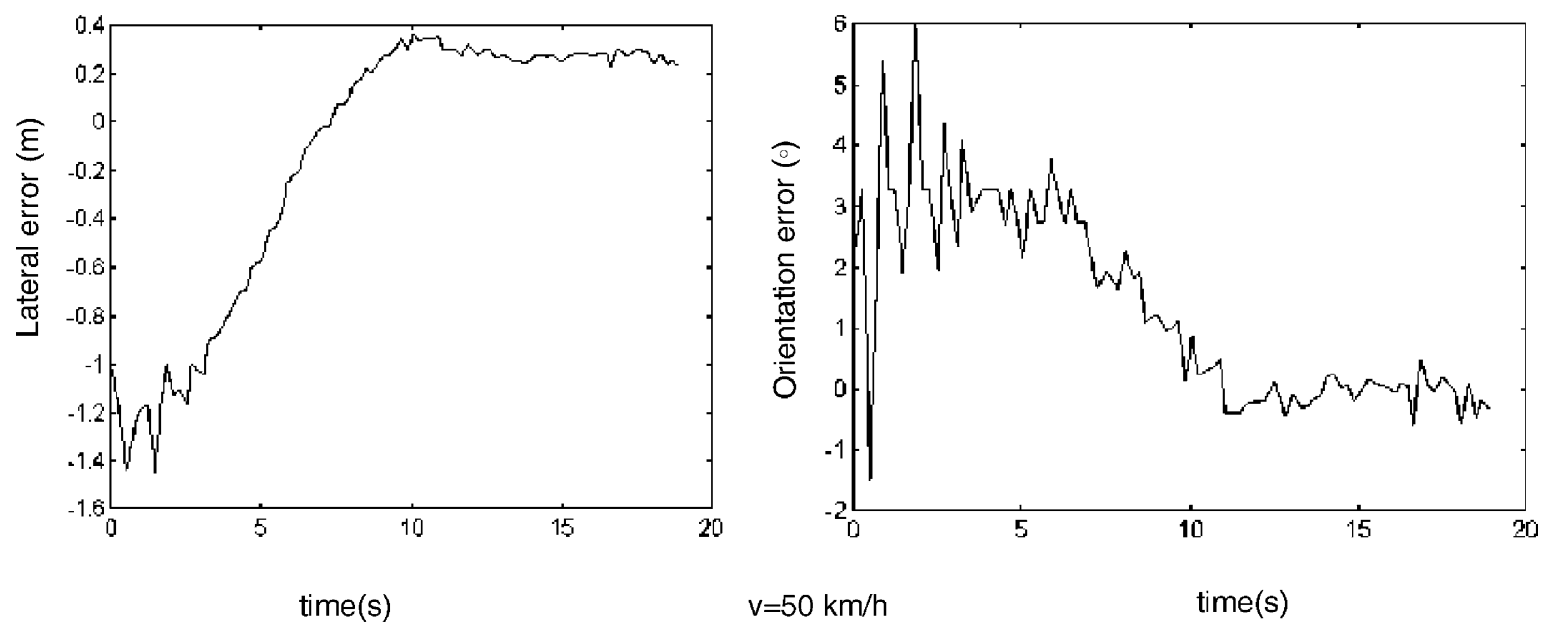

Fig. 7. Transient response of the lateral and orientation error for $v=50 \mathrm{~km} / \mathrm{h}$. 


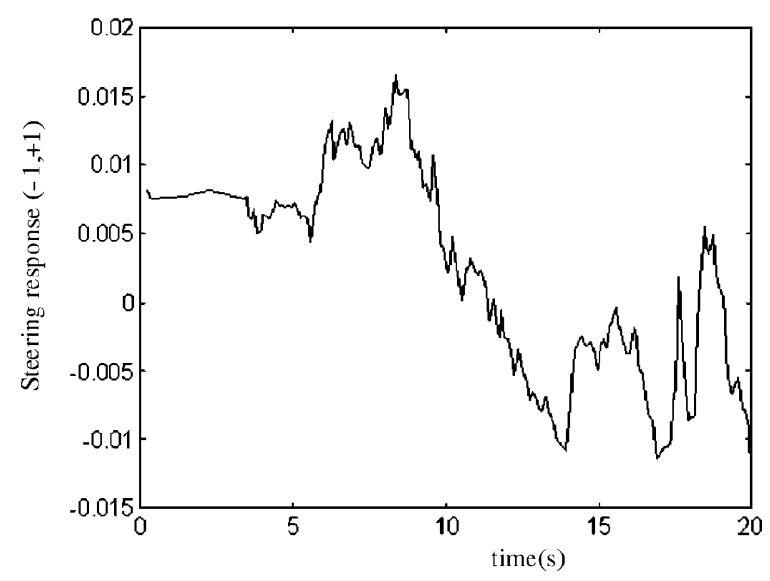

Fig. 8. Steering normalized response (between -1 and +1 ) provided by the lateral controller for $v=50 \mathrm{~km} / \mathrm{h}$.

accelerator to the velocity controller in order to keep a reference speed of $20 \mathrm{~km} / \mathrm{h}$. The comparison is graphically depicted in Fig. 9.

On one hand, one can observe how the human driver takes less time than the automatic controller to achieve lateral and orientation errors close to zero. On the other hand, the steady-state errors are similar in both cases. Surprisingly, human driving turns out in sporadic separations from the reference trajectory up to $40-50 \mathrm{~cm}$, without incurring in dangerous behavior, while the automatic controller keeps the vehicle under lower lateral error values once stabilized. Far from being an isolated fact, this circumstance was repeatedly observed in several practical experiments. As conclusion, the lateral control law developed in this work can reasonably be considered to be valid to drive an Ackerman-like vehicle as precisely as a human can. During the last year, Babieca ran over hundreds of kilometers in lots of successful autonomous missions carried out along the test circuit using the non-linear control law described in this paper. A live demonstration exhibiting the system capacities on autonomous driving using the non-linear control law described in this paper was carried out during the IEEE Conference on Intelligent Vehicles 2002, in a private circuit located at Satory (Versailles), France. A complete set of video files demonstrating the
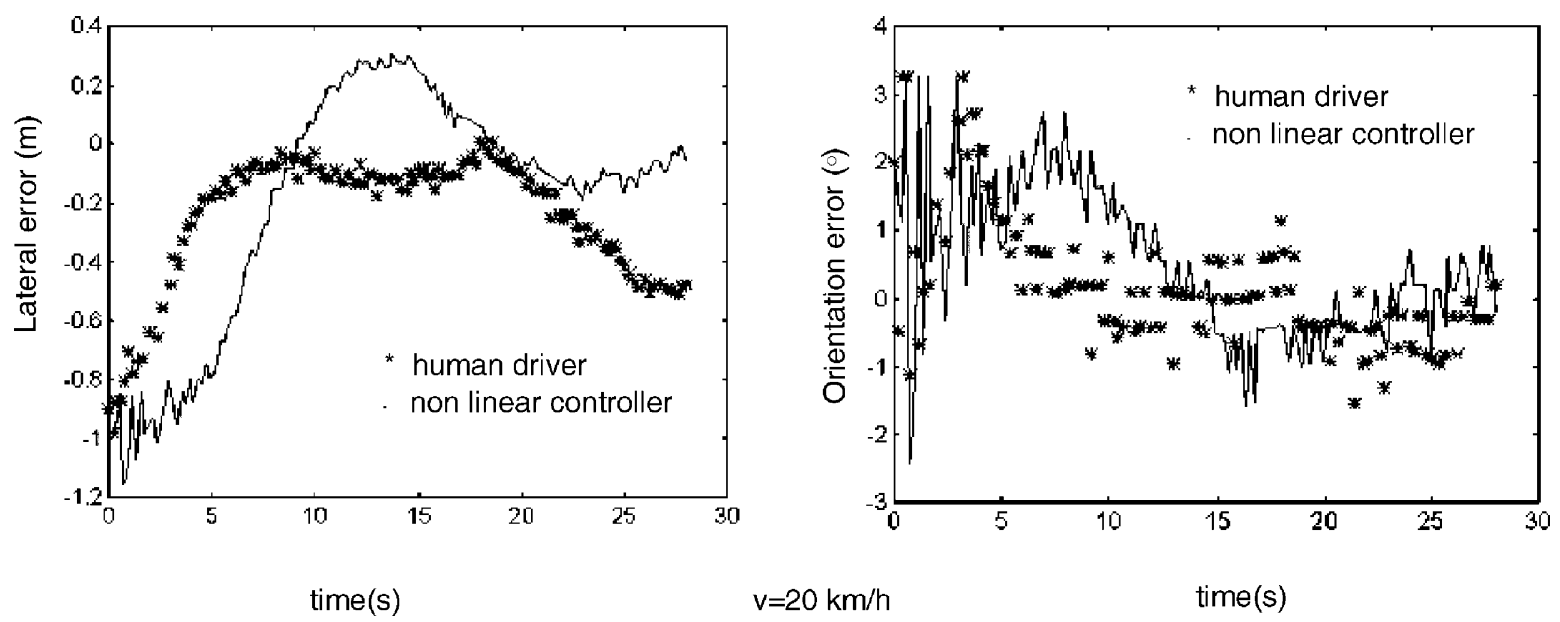

Fig. 9. Comparison between automatic guidance and human driving at $v=20 \mathrm{~km} / \mathrm{h}$. 
operational performance of the control system in real test circuits (both in Arganda del Rey and in Satory) can be retrieved from ftp://www.depeca.uah.es/pub/vision.

\section{Conclusions}

To conclude, the next key points should be remarked.

- First of all, the non-linear control law described in this work has proved its analytical and empirical stability for lateral driving of Ackerman-like vehicles. In fact, it has been implemented on a real commercial vehicle slightly modified so as to allow for autonomous operation, and tested on two different private circuits.

- Vehicle commanded actuation is taken into account by considering the current velocity in the design of the controller coefficients. This permits to adapt the steering angle as a function of driving conditions.

- As demonstrated in practical trials, driving precision achieved in steady-state by the lateral control law is as accurate as that of a human driver under normal conditions. However, human drivers provide quite a faster response in transients as they use a feed-forward control component initially. Accordingly, as a future work, a similar approach (feed-forward component based) will be undertaken in order to improve the transient response of the nonlinear lateral controller described in this paper.

Nonetheless, in spite of having achieved some promising results there is still much space for improvement concerning vehicle stability and oscillations. Indeed, our current work focuses on the development of more precise vehicle models accounting for actuator dynamics and non-linearities. Thus, a new non-linear control law should be developed in an attempt to increase stability and comfortability when driving at high speed.

\section{Acknowledgements}

This work has been funded by the Department of Electronics of the UAH (Universidad de Alcalá), and the generous support of the Industrial Automation Institute of the CSIC (Consejo Superior de Investigaciones Cientificas).

\section{References}

[1] J. Ackermann, W. Sienel, Robust control for automated steering, in: Proceedings of the 1990 American Control Conference, ACC' 90 , San Diego, CA, 1990, pp. 795-800.

[2] J. Ackermann, J. Guldner, W. Sienel, R. Steinhauser, V.I. Utkin, Linear and nonlinear controller design for robust automatic steering, in: Proceedings of the IEEE Transactions on Control Systems Technology, vol. 3, No. 1, 1995.

[3] M. Barabanov, A Linux-based real-time operating system, Master of Science Thesis in Computer Science, New Mexico Institute of Mining and Technology, New Mexico, June 1997.

[4] A. Broggi, M. Bertozzi, A. Fascioli, G. Conte, Automatic Vehicle Guidance: The Experience of the ARGO Autonomous Vehicle, World Scientific, Singapore, 1999.

[5] R.H. Byrne, C.T. Abdallah, P. Dorato, Experimental results in robust lateral control of highway vehicles, IEEE Control Systems 18 (2) (1998) 70-76.

[6] S. Cameron, P. Proberdt, Advanced Guided Vehicles, Aspects of the Oxford AGV Project, World Scientific, Singapore, 1994.

[7] L. Cordesses, P. Martinet, B. Thuilot, M. Berducat, GPS-based control of a land vehicle, in: Proceedings of the IAARC/IFAC/IEEE International Symposium on Automation and Robotics in Construction, ISARC'99, Madrid, September 22-24, 1999.

[8] T. De Pedro, R. Garcia, C. Gonzalez, J.E. Naranjo, J. Reviejo, M.A. Sotelo, Vehicle automatic driving system based on GNSS, in: Proceedings of the International Conference on Intelligent Vehicles, Seville, 2001.

[9] E.D. Dickmanns, B.D. Mysliwetz, Recursive 3D road and relative ego-state recognition, in: Proceedings of the IEEE Transactions on Pattern Analysis and Machine Intelligence, vol. 14, No. 2, February 1992.

[10] U. Franke, D. Gavrila, S. Gorzig, F. Lindner, F. Paitzold, C. Wohler, Autonomous driving goes downtown, in: Proceedings of the IEEE Intelligent Vehicles Symposium, Stuttgart, Germany, 1998. 
[11] R. Gregor, M. Lutzeler, M. Pellkofer, K.H. Siedersberger, E.D. Dickmanns, EMS-vision: A perceptual system for autonomous vehicles, in: Proceedings of the IEEE Transactions on Intelligent Transportation Systems, vol. 3, No. 1, March 2002.

[12] J. Guldner, W. Sienel, H. Tan, J. Ackermann, S. Patwardhan, T. Bunte, Robust automatic steering control for look-down reference systems with front and rear sensors, in: Proceedings of the IEEE Transactions on Control Systems Technology, vol. 7, No. 1, 1999.

[13] T. Hessburg, M. Tomizuka, Fuzzy logic control for lateral vehicle guidance, IEEE Control System Magazine 14 (1994) 55-63.

[14] J. Kosecka, R. Blasi, C.J. Taylor, J. Malik, Vision-based lateral control of vehicles, in: Proceedings of the Intelligent Transportation Systems Conference, Boston, 1997.

[15] J. Luo, P. Tsiotras, Control design for systems in chained form with bounded inputs, in: Proceedings of the American Control Conference, Philadelphia, PA, June 24-25, 1998.

[16] H. Peng, M. Tomizuka, Lateral control of front-wheel steering rubber-tire vehicles, Technical Report No. UCB-ITS-PRR-90-5, Institute of Transportation Studies, Berkeley, CA, 1990.

[17] M.A. Sotelo, F.J. Rodriguez, L. Magdalena, L.M. Bergasa, L. Boquete, A colour vision-based lane tracking system for autonomous driving on unmarked roads, Autonomous Robots 16 (1) (2004) in press.

[18] H. Sussmann, E. Sontag, Y. Yang, A general result on the stabilization of linear systems using bounded controls, IEEE Transactions on Automatic Control 39 (12) (1994) 2411-2425.

[19] C.J. Taylor, J. Kosecka, R. Blasi, J. Malik, A comparative study of vision-based lateral control strategies for autonomous highway driving, The International Journal of Robotics Research 18 (5) (1999) 442-453.

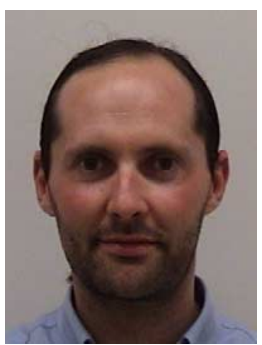

Miguel Angel Sotelo received his Dr. Ing. degree in electrical engineering in 1996 from the Technical University of Madrid, and his Ph.D. degree in electrical engineering in 2001 from the University of Alcalá, Alcalá de Henares, Madrid, Spain. From 1993 to 1994 he has been a researcher at the Department of Electronics, University of Alcalá, where he is currently an Associate Professor. His research interests include real-time computer vision and control systems for autonomous and assisted intelligent vehicles. He has been recipient of the Best Research Award in the domain of Automotive and Vehicular Applications in Spain, in 2002. He is the author of more than 50 refereed publications in international journals, book chapters, and conference proceedings. 